\title{
DYNAMICS OF DISSOLUTION OF GAS BUBBLES OR POCKETS IN TISSUES*
}

\author{
WEN-JEI YANG $\dagger$ and C. Y. LIANG $\ddagger$ \\ Department of Mechanical Engineering, The University of Michigan, Ann Arbor, Michigan 48 104, U.S.A.
}

\begin{abstract}
A mathematical model is developed which describes the dynamic characteristics of gas bubbles in subcutaneous tissues. Consideration is given to both inert and reacting gases. The effects of blood perfusion (or oxygen consumption in the tissue in dead animals), diffusion of the dissolved gases, a creep process occuring in the tissue, and thermodynamic behavior of the gases in the cavity on the dissolution of the gas bubbles are taken into account. The stressstrain relation of the tissues in creep is described by the standard linear model of viscoelasticity. The theoretical analysis is presented in three categories: (i) tissue creep controlled, (ii) mass transfer controlled and (iii) the intermediate case where both mechanisms are of comparable importance. A close agreement is obtained between the theoretical predictions and the existing experimental data for subcutaneous inert gas pockets in air breathing rats.
\end{abstract}

\section{INTRODUCTION}

Evolution of gas bubbles in tissues and other parts of the human body may occur on rapid decompression. It has been found that formation of bubbles normally occurs in great abundance in the fatty tissues (Harvey et al., 1944a, 1944b; Harvey, 1945). The survey of the literature pertinent to the evolution of bubbles on decompression is available (Chan and Yang, 1969). The gas bubbles evolved following rapid decompression are mostly nitrogen or air (the mixture of nitrogen and oxygen) that has been dissolved in the body under high pressure. However, the formation of carbon dioxide bubbles has been found in tissues after muscular activity following decompression. It is reported that breathing oxygen helps the removal of nitrogen bubbles (to reduce the incidence of 'bends' and 'chokes').

Sometimes, gas bubbles are purposely injected into subcutaneous tissues, liver and perirenal fat. Quantitative data obtained with the subcutaneous gas pocket, an in vivo tonometry system, provide basic information concerning tissue-capillary gas exchange: specifically for the determinations of-(i) permeation of the dissolved gases in the tissues and their interaction with hemoglobin (Dale and Rahn, 1952; Rahn and Canfield, 1955; Van Liew, 1955; Piiper, Canfield and Rahn, 1962; Piiper, Hamphrey and Rahn, 1962; Tobin et al., 1962; Van Liew, 1962b; Piiper, 1963; Tucker and Tenney, 1966; Van Liew and Passke, 1967; Van Liew, 1968a, b); (ii) tension of the dissolved gases in the tissues in the constant composition state (Van Liew, 1962a, b, c, 1968b); and (iii) the influx and efflux of inert gases across the surface of a decomposition bubble (Van Liew et al., 1965, 1968/1969; Van Liew and Hlastala, 1969).

The experimental study of tissue-capillary gas exchange using a subcutaneous gas pocket in rats is generally performed in two stages: preparatory and test stages (for example, Van Liew, 1955; Piiper et al., 1962; Tucker and Tenney, 1966; Van Liew, 1968a). In the preparatory stage, air-filled gas pockets (of 20-30 ml) were maintained for several days

*Received 23 August 1971.

†Professor.

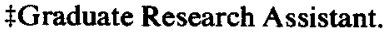


(from 5-30 days depending on investigators) during which time the local tissue reaction subsides. By the fifth day, the measured $P_{\mathrm{O}_{2}}$ and $P_{\mathrm{CO}_{2}}$ of pocket gases had reached reasonably constant values, indicating that blood perfusion to the pocket had stabilized. For the following 3 weeks such pockets are in a state of constant composition of $\mathrm{O}_{2}$ and $\mathrm{CO}_{2}$ and were satisfactory for use to study the disappearance of test gases.

In the test stage, $20 \mathrm{ml}$ of test gas were injected into the pocket after evacuation of the preparatory air. In about $2 \mathrm{hr}$ after the introduction of the foreign gas, the $\mathrm{O}_{2}$ and $\mathrm{CO}_{2}$ composition of the pocket will be constant and remain so until all the gas has been absorbed (Van Liew, 1955). The subsequent time course of the volume of this pocket was then determined by extracting the gas from the pocket into a calibrated syringe, noting the volume and time, and then re-injecting the gas from the syringe back into the pocket. The subcutaneous gas pockets containing the foreign gas-air, inert gases such as argon, helium, nitrogen, hydrogen, cyclopropane $\left(\mathrm{C}_{3} \mathrm{H}_{6}\right)$, nitrous oxide $\left(\mathrm{N}_{2} \mathrm{O}\right)$ and sulfur hexafluoride $\left(\mathrm{SF}_{6}\right)$, or reacting gases such as oxygen, carbon monoxide and carbon dioxide -were tested in air-breathing, oxygenbreathing or dead rats.

Depending on the physical characteristics of the foreign gas and the breathing environment, the migration of a single gas or two gases may occur across the pocket-tissue interface. The single-gas case is characterized by the flux of any gas in an oxygen-breathing animal, or of nitrogen in an air-breathing animal. If instead of nitrogen, some other gas (inert or reacting gas including air) is injected into the pocket, and if the animal breathes atmospheric air, the pocket will soon contain two gases, nitrogen from the blood and the foreign gas injected. This corresponds to the two-gas case in which the fluxes of both gases must be reckoned with. These two fluxes are interdependent, that is, the efflux of either one alters the driving pressure gradient of the other. If the foreign inert gas such as $\mathrm{SF}_{6}$ is nearly insoluble, the pocket volume will increase substantially over initial volume before it begins to decrease toward zero. On the other hand, a pocket of highly soluble inert gas such as $\mathrm{N}_{2} \mathrm{O}$ will decrease rapidly, even before more than a small quantity of nitrogen can enter.

The dynamics of a gas cavity in a tissuecapillary system may be considered as a problem of determining the distribution of the dissolved-gas tension under the action of the difference in pressure in the tissue at the interface and at a point a large distance from: the gas cavity. The gas tension in the tissue at the interface depends on the pressure exerted by the gas in the cavity. Any external force variation may be imposed on the tissue through muscular activity. However, it will induce a creep process in the system which, in turn, affects the gas pressure of the cavity and consequently its difference with the gas tension in the tissue at a large distance from the cavity. It is the pressure difference which will induce the migration of the dissolved gas to or from the cavity. Hence, there is a coupling between the equation of motion (or stress equilibrium equation) and the equation for the pressure (or concentration) field in the tissue-capillary system.

Piiper et al. (1962) have analyzed the gas exchange between a subcutaneous gas pocket and the tissue-capillary system for the singlegas diffusion case by treating the tissuecapillary system as a lumped-parameter unit. The work was extended to the two-gas diffusion case by Tucker and Tenney (1966). Van Liew (1968a) has given a theoretical analysis for both the single- and two-gas diffusion cases by treating the tissue-capillary system as a distributed-parameter unit. The expression predicting the volume history of the gas pocket is obtained by solving the steady one-dimensional pressure distribution in the tissue-capillary system (which is treated as an infinite slab) followed by equating the rate of gas exit from the pocket 
to the rate of gas uptake in the tissuecapillary system. Both the rate of blood perfusion in the tissue in living animals and that of metabolism or oxygen consumption in the tissue in dead animals are considered constant.

As shown by Piiper et al. (1962), Tucker and Tenney (1966) and Van Liew (1968), total gas pressure in subcutaneous gas pockets is essentially atmospheric and $2 \mathrm{hr}$ after the introduction, the gas composition of the pocket will be constant and remain so until all the gas has been absorbed. Thus, a constant total pressure difference for any particular foreign gas is established throughout the course of the gas absorption. The creep effect of the tissue-capillary environment may thus be neglected and the volume history of the gas pocket is controlled by the rate of mass transfer between the tissue-capillary system and the pocket wall.

On the other hand, conditions may be such that mass transfer effects on the volume change of a gas cavity are minor compared with the creep process of the tissue when an external force variation is impressed on the system. Muscular activity is an example. Since mass transfer across the pocket wall due to the total pressure difference is insignificant, the gas or gas mixture confined in the pocket may be viewed to undergo a reversible polytropic process. However, no study on the creep controlling case has been reported as far as the authors are aware.

In the present paper, a mathematical model is developed which describes the dynamic behavior of a gas bubble or pocket situated in homogeneous and isotropic tissues. The dynamic equation of a gas cavity in the tissue under a creep process is derived using the standard linear model of viscoelasticity to describe the stress-strain relation. For the case where mass transfer from the pocket to the tissue-capillary system is the controlling mechanism, the bubble dynamic equations are derived for both the inert and reacting gases including the effects of diffusion, perfusion and thermodynamic behavior of the cavity gases. The analysis is then extended to the general case in which the contributions of both mass transfer and creep are of comparable importance. Theoretical results are compared with the experimental data for subcutaneous inert gas pockets in air-breathing animals (Tucker and Tenney, 1966).

\section{ANALYSIS}

Consider a spherical gas cavity of radius $R_{o}$ (mean radius if non-spherical) situated in a homogeneous and isotropic tissue-capillary system with the dissolved gas of uniform tension $P_{\infty}$. Initially, the total gas pressure in the cavity is $P_{g o}$, which is in equilibrium with the surface tension $2 \sigma / R_{o}$ and the uniform shear stress in the system $\tau_{\infty}(0)$. An external load $\tau_{\infty}(t)$ is then impressed on the system at a large distance from the cavity. At any time $t>0$, the gas pressure in the cavity is $P_{g}$ and the dissolved-gas tension and the shear strain in the tissue-capillary system are $P$ and $\epsilon_{r r}$, respectively. Due to the variations in the pressure difference $\left(P_{g}-P_{\infty}\right)$ and strain rate $\dot{\boldsymbol{\epsilon}}_{r r}$, the cavity may grow or shrink depending on the nature of the gas, of the tissue-capillary system, and of the stress applied on the cavity wall. The growth or collapse of the gas cavity is accompanied by the migration of the gas across the cavity wall. It is postulated that both the air cavity and the tissue-capillary system remain at the same temperature $T$ until all the gas has been absorbed.

\section{Bubble Dynamics caused by the Creep of the Tissue only}

For analytical convenience, a spherical polar coordinate system $(r, \theta, \phi)$ is used. Its origin is fixed at the center of the gas bubble whose radius is $R$ at any time $t>0$. If the tissue is homogeneous and isotropic, the distribution of the stresses around the bubble will be spherically symmetrical. Then, the non-diagonal components of the stress tensor are equal to zero, $\tau_{r r}=-2 \tau_{\theta \theta}=-2 \tau_{\phi \phi}$ and the effective stress is $\tau_{r r}-\tau_{\theta \theta}$. The stress-strain 
relation for standard linear model reads

$$
(1+\lambda D)\left(\tau_{r r}-\tau_{\theta \theta}\right)=\eta(1+\mu D) \epsilon_{r r},
$$

where $\lambda, \eta$ and $\mu$ are constants determined by the properties of the tissue, the temperature etc. This model is used in reference to Frankel and Burstein (1970). However, the generalized Maxwell or Kelvin model can also be used without much complexity in the subsequent analysis, see Yang and Liang (1971). The creep rate is defined as

$$
\dot{\epsilon}_{r r}=\partial v_{r} / \partial r
$$

where $v_{r}$ is the radial component of the deformation rate tensor $\vec{v}$. The continuity equation is

$$
\operatorname{div} \vec{v}=\frac{\partial v_{r}}{\partial r}+\frac{2 v_{r}}{r}=0
$$

The stresses $\tau_{r r}$ and $\tau_{\theta \theta}$ must satisfy the equilibrium equation

$$
\frac{\partial \tau_{r r}}{\partial r}+\frac{2\left(\tau_{r r}-\tau_{\theta \theta}\right)}{r}=0
$$

with boundary conditions

$$
\begin{aligned}
& \tau_{r r}(R, t)=-P_{g}+2 \sigma / R, \\
& \tau_{r r}(\infty, t)=\tau_{\infty}(t),
\end{aligned}
$$

where $P_{g}$ is the gas pressure inside the bubble and $\sigma$ is the coefficient of surface tension.

The integration of equation (3) from $r=R$ to $r=r$ yields

$$
v_{r}=(R / r)^{2} \dot{R},
$$

where the superscript - denotes the time derivative. Following the substitution of equation (6), equation (2) is integrated with respect to time from $t=0$ to $t=t$. It gives

$$
\epsilon_{r r}=\frac{2}{3 r^{3}}\left(R_{o}^{3}-R^{3}\right)+\epsilon_{r r o}
$$

where $\epsilon_{\text {rro }}$ is the initial local strain. Taking equations (1) and (7) into account, equation (4) is solved subject to the boundary conditions (5). One then gets the dynamic equation of a gas bubble in tissues

$$
\begin{aligned}
& \tau_{\infty}+P_{g}-\frac{2 \sigma}{R}+\lambda\left(\dot{\tau}_{\infty}+\dot{P}_{g}-\frac{2 \sigma \dot{R}}{R^{2}}\right)+\Delta \tau_{r r} \\
& =\frac{4 \eta}{9 R^{3}}\left(R^{3}-R_{o}{ }^{3}+3 \mu R^{2} \dot{R}\right)
\end{aligned}
$$

subject to the initial conditions

$$
\begin{aligned}
& R(0)=R_{o}, \dot{R}(0)=0, \tau_{\infty}(0)=-P_{g o}+\frac{2 \sigma}{R_{o}} \\
& \dot{\tau}_{\infty}(0)=0, P_{g}(0)=P_{g o}, \dot{P}_{g}(0)=0
\end{aligned}
$$

where $\Delta \tau_{r r o}=-\tau_{r r}\left(R_{o}, 0\right)+\tau_{r r}(\infty, 0)$ and $\tau_{\infty}$ is identical with $\tau_{r r}(\infty, t)$. If the gas in the cavity undergoes a reversible polytropic process the variations of its pressure and volume can be related as

$$
P_{g}(t)=P_{g o}\left(R / R_{o}\right)^{3 \gamma} .
$$

Here $\gamma$ is the polytropic exponent and takes the value of unity for isothermal process. The combination of equations (8a) and (10) yields

$$
\begin{aligned}
\tau_{\infty} & +P_{g o}\left(\frac{R}{R_{o}}\right)^{3}-\frac{2 \sigma}{R} \\
& +\lambda\left[\dot{\tau}_{\infty}+3 P_{g o}\left(\frac{R}{R_{o}}\right)^{3} \frac{\dot{R}}{R}-\frac{2 \sigma \dot{R}}{R^{2}}\right]+\Delta \tau_{r r o} \\
& =\frac{4 \eta}{9 R^{3}}\left(R^{3}-R_{o}{ }^{3}+3 \mu R^{2} \dot{R}\right)
\end{aligned}
$$

\section{Bubble Dynamics caused by Mass. Transfer only}

In the diffusion of a gas out of decompression bubbles or artificially injected gas pockets, the gas diffusing through living tissues meets blood capillaries at various depths. Either the solution of the gas in blood or its chemical reaction with blood elements has created multiple small sinks for the gas within the diffusion barrier. Thus, the dissolved gas concentration decreases with distance from the gas-tissue interface because 
of two factors: (i) blood leaving capillaries removes the gas from the system-mass consumption and (ii) divergence of radii of the sphere of tissue around the bubble or pocket-mass diffusion. The difference between the rates of mass diffusion and mass consumption should be equal to the time-rate change in the amount of locally dissolved gas according to the principle of mass conservation.

\section{a. Inert gases}

For inert gases such as nitrogen, argon, helium, hydrogen, nitrous oxide and sulfur hexafluoride which are not metabolized and which do not enter into chemical reaction with blood elements, disappearance of gas from the diffusion system will be by solution in blood. The rate of disappearance is directly proportional to the difference between the dissolved gas pressure $P$ and that in the arterial blood $P_{\infty}$ (Van Liew, 1968). Thus, the diffusion equation reads

$$
\frac{\partial P}{\partial t}=\frac{\mathscr{D}}{r^{2}} \frac{\partial}{\partial r}\left(r^{2} \frac{\partial P}{\partial r}\right)-A\left(P-P_{\infty}\right),
$$

where $\mathscr{D}$ is the mass diffusion coefficient of the dissolved gas in the tissue, $A=\alpha_{b} k Q / \alpha_{t}$, $\alpha_{b}$ is the gas solubility in blood, $\alpha_{t}$ the gas solubility in tissue, $k$ the coefficient of end capillary saturation, and $Q$ is the rate of actual blood perfusion. $k Q$ is the effective blood perfusion. The appropriate initial and boundary conditions are

$$
\begin{aligned}
& P(r, 0)=P_{\infty} \\
& P(R, t)=P_{g}, P(\infty, t)=P_{\infty} .
\end{aligned}
$$

Now, a solution of the diffusion problem which is valid only for a stationary bubble boundary is to be obtained as a reasonable physical approximation for a slowly moving interface between a gas phase and a solid mass of living tissue. The accuracy of the approximation has been found to be quite good for the diffusion of a gas bubble in liquid-gas solutions (Epstein and Plesset, 1950; Yang, 1971).

Through the transformation of

$$
u=r\left(P-P_{\infty}\right) \text { and } \xi=r-R
$$

equation (11) becomes

$$
\frac{\partial u}{\partial t}=\mathscr{D} \frac{\partial^{2} u}{\partial \xi^{2}}-A u
$$

with the initial and boundary conditions

$$
\begin{aligned}
& u(\xi, 0)=0 \\
& u(0, t)=R\left(P_{g}-P_{\infty}\right) .
\end{aligned}
$$

The problem now is reduced to a familiar one in heat conduction, the solution of which is available in Carslaw and Jaeger (1959).

$$
\begin{aligned}
u(\xi, t)= & R\left(P_{g}-P_{\infty}\right)[\exp (-A t) \\
& \times \operatorname{erfc} \frac{\xi}{(4 \mathscr{D} t)^{1 / 2}}+1-\exp (-A t) \\
& -\int_{0}^{t} A \exp \left(-A t^{\prime}\right) \\
& \left.\times \int_{0}^{\xi} \frac{\exp \left(-\xi^{2} / 4 \pi t^{\prime}\right)}{\left(\pi \mathscr{D} t^{\prime}\right)^{1 / 2}} \mathrm{~d} \xi^{\prime} \mathrm{d} t^{\prime}\right] .
\end{aligned}
$$

The quantity of interest is the pressure gradient at $r=R$, which is found to be

$$
(\partial P / \partial r)_{r=R}=\left(P_{g}-P_{\infty}\right) I_{1}(t),
$$

where

$$
I_{1}(t)=\frac{1}{R}+\frac{\exp (-A t)}{(\pi \mathscr{D} t)^{1 / 2}}+(A / \mathscr{D})^{1 / 2} \operatorname{erf}(A t)^{1 / 2}
$$

At large times, $I_{1}(t)$ approaches the value

$$
I_{1}(t)=\frac{1}{R}+\left(\frac{A}{\mathscr{D}}\right)^{1 / 2} \text {. }
$$

\section{b. Reacting gases}

The reacting gases include oxygen, carbon monoxide and carbon dioxide. The diffusion and perfusion of carbon dioxide will not be 
considered here due to complexity resulting from chemical combination with blood elements, blood perfusion or metabolism. Consideration is given only to the following three cases: (i) oxygen in dead animalthe gas diffuses out and disappears due to utilization by the tissue; (ii) carbon monoxide - the gas diffuses out and disappears by combining with hemoglobin in the blood perfusing in the tissue; and (iii) oxygen in living animal the gas disappears both by metabolism and by carriage in blood. The diffusion equation applicable to all these three cases may be expressed as

$$
\frac{\partial P}{\partial t}=\frac{\mathscr{D}}{r^{2}} \frac{\partial}{\partial r^{2}}\left(r^{2} \frac{\partial P}{\partial r}\right)-B,
$$

where $B=q / \alpha_{t}$ for case (i) and $B=Q \Delta C / \alpha_{b}$ for cases (ii) and (iii). In equation (19), $q$ is the rate of oxygen consumption in the tissue and $\Delta C$ is the change of the dissolved gas content in blood. The appropriate initial and boundary conditions are identical with equation (12), in which $P_{\infty}=0$ in cases (i) and (ii) and $P_{\infty}=40 \mathrm{~mm} \mathrm{Hg}$ in case (iii).

Equation (19) is solved in a manner exactly analogous to the previous section. One finds

$$
\begin{aligned}
u(\xi, t)= & R\left(P_{g}-P_{\infty}\right) \\
& \times\left[\left(1+B^{*} t+\frac{B^{*} \xi^{2}}{2 \mathscr{D}}\right) \operatorname{erfc} \frac{\xi}{2(\mathscr{D} t)^{1 / 2}}\right. \\
- & \left.B^{*} \xi\left(\frac{t}{\pi \mathscr{D}}\right)^{1 / 2} \exp \left(-\frac{\xi^{2}}{4 \mathscr{D} t}\right)-B^{*} t\right]
\end{aligned}
$$

in which

$$
B^{*}=B /\left(P_{g}-P_{\infty}\right) .
$$

Hence the pressure gradient at $r=R$ is

$$
\left(\frac{\partial P}{\partial r}\right)_{r=R}=\left(P_{\infty}-P_{g}\right) I_{2}(t)
$$

where

$$
\begin{aligned}
I_{2}(t)= & \frac{1}{R}+\frac{1}{(\pi \mathscr{D} t)^{1 / 2}} \\
& +B^{*} t\left[\frac{1}{R}+\frac{2}{(\pi \mathscr{D} t)^{1 / 2}}\right]
\end{aligned}
$$

At large times $I_{2}$ approaches

$$
I_{2}(t)=\left(1+B^{*} t\right) / R .
$$

If there is no externally applied force on the tissue and if the total pressure inside the gas bubble does not vary appreciably over a period of time, mass transfer is the mechanism controlling the dynamic behavior of the bubble. Problems of this kind can be further classified into two categories, i.e. (i) single-gas diffusion, and (ii) multi-gas diffusion.

(i) Single-gas diffusion case. For a pocket of nitrogen, in an air-breathing animal or for any inert gas in an animal breathing oxygen, the volume change of the pocket is determined by single gas diffusion. If the gas inside the pocket behaves like an ideal gas, its equation of state is

$$
4 \pi R^{3} P_{g} / 3=m \bar{R} T,
$$

where $\bar{R}$ is the gas constant. For small variation in $\boldsymbol{P}_{g}$, differentiation of the above equation with respect to time yields

$$
\dot{R}=\frac{\dot{m} \bar{R} T}{4 \pi R^{2} P_{g}} .
$$

The rate of mass diffusion $m$ can be related to the pressure gradient in tissue at the bubble surface by Fick's Law by the expression

$$
\dot{m}=4 \pi R^{2} \mathscr{D} H(\partial P / \partial r)_{r=R},
$$

where $H=\alpha_{t} / \rho$ and $\rho$ is the gas density in the pocket. By substituting equation (24) into equation (23), one gets the bubble dynamic equation for the single-gas diffusion case.

$$
P_{g} \dot{R}=\mathscr{D} H \bar{R} T(\partial P / \partial r)_{r=R}
$$

or

$$
P_{g} \dot{R}=\mathscr{D H} \bar{R} T\left(P_{\infty}-P_{g}\right) I_{i} \quad(i=1,2)
$$


subject to the initial condition $R(0)=R_{o}$. The volume history of the pocket may be obtained by the numerical integration of equations (18) and (25b).

(ii) Multi-gas diffusion case. If instead of nitrogen, some inert gas such as argon, hydrogen, helium, sulfur hexafloride, cyclopropane or nitrous oxide is initially injected into the pocket, and if the animal breathes normal atmospheric air, two gases will cross the pocket wall, nitrogen from the blood and the foreign inert gas injected. In that case, the relative rate of movement of the two gases determines the volume history of the gas pocket. If each gas behaves ideally in the pocket, then each gas component satisfies the equation of state

$$
P_{g j}=\frac{3 m_{j} \bar{R}_{j} T}{4 \pi R^{3}}
$$

where the subscript $j$ denotes the $j$-th gas component. Since Dalton's Law requires that

$$
P_{g}=\sum P_{g j}=\frac{3 T}{4 \pi R^{3}} \sum m_{j} \bar{R}_{j}
$$

therefore, the rate of mass diffusion $m_{j}$ can be expressed as

$$
\dot{m}_{j}=4 \pi R^{2} \mathscr{D}_{j} H_{j}\left(\partial P_{j} / \partial r\right)_{r=R} .
$$

By differentiating equation (27) with respect to time followed by the elimination of $\dot{m}_{j}$ from the resulting expression and equation (28), one gets the bubble dynamic equation for the multi-gas diffusion case.

$$
P_{g} \dot{R}=T \sum \mathscr{D}_{j} H_{j} \bar{R}_{j}\left(\partial P_{j} / \partial r\right)_{r=R}
$$

or

$$
P_{g} \dot{R}=T \sum \mathscr{D}_{j} H_{j} \bar{R}_{j}\left(P_{\infty}-P_{g}\right)_{j} I_{i}
$$

It should be noted that since diffusion of nitrogen and the inert gas is taking place simultaneously, the partial pressure of each gas $P_{g j}$ in equation (29b) varies with time. $P_{g j}$ can be determined, however, by integrating $\dot{m}$ in equation (28) with respect to time from $t=0$ to $t=t$ and by substituting $m_{i}$ thus obtained into equation (28). The expression is founded to be

$$
\begin{aligned}
P_{g j}= & \frac{3 \bar{R}_{j} T}{4 \pi R^{3}} \\
& \times\left[4 \pi \mathscr{D}_{j} H_{j} \int_{0}^{t} R^{2}\left(\partial P_{j} / \partial r\right)_{r=R} \mathrm{~d} t+m_{o j}\right],
\end{aligned}
$$

where $m_{o j}$ denotes the initial mass of the $j$-th gas in the bubble. Numerical result can be obtained by solving equations (18) and (29) and (30) simultaneously with the use of a digital computer.

\section{Bubble Dynamics caused by both Creep and Mass Transfer}

This is the general case in which both the creep and mass transfer processes in the tissue-capillary system affect the growth and shrinkage of the gas cavity. The volume history of the bubble can be obtained by simultaneously solving equations (8a), (25b) or (29b) subject to the initial conditions (9) using a computing machine.

COMPARISON OF THEORETICAL RESULTS WITH EXPERIMENTAL DATA FOR MASS TRANSFER CONTROLLING CASE

Theoretical results were obtained for the mass transfer controlling case - the inert gas exchange in subcutaneous gas pockets of airbreathing animals using the physical and physiological data listed in Table 1 (Bartels and Opitz, 1958; Van Liew, 1968a). $Q$ is $0.33 \mathrm{~mm}$ of blood $/ \mathrm{min}$ per $\mathrm{cm}^{3}$ of tissue. The value of $k$, the coefficient of end-capillary

Table 1. Physical and physiological properties used in calculations $\left(\alpha_{b}=\alpha_{t}=\alpha\right)$

\begin{tabular}{lccc}
\hline Gas & $\begin{array}{c}10^{5}, \alpha \\
\left(\mathrm{ml} \mathrm{m}^{-1},\right. \\
\left.\mathrm{mm} \mathrm{Hg}^{-1}\right)\end{array}$ & $\begin{array}{c}\text { Diffusivity } \times \\
\text { solubility } \times 10^{8}, \\
\left(\mathrm{~cm}^{2} . \mathrm{min}^{-1}\right. \\
\left.\mathrm{mm} \mathrm{Hg}^{-1}\right)\end{array}$ & $\begin{array}{c}\text { Effective } \\
\text { perfusion } \\
\text { rate, } k Q \\
\left(\mathrm{~min}^{-1}\right)\end{array}$ \\
\hline $\mathrm{N}_{2}$ & 1.84 & 1.00 & 0.26 \\
$\mathrm{~A}$ & 4.1 & 1.87 & 0.223 \\
$\mathrm{H}_{2}$ & 2.12 & 1.94 & 0.33 \\
$\mathrm{He}$ & 1.25 & 1.44 & 0.33 \\
$\mathrm{SF}_{6}$ & 0.606 & 0.145 & 0.13 \\
$\mathrm{~N}_{2} \mathrm{O}$ & 68.0 & 29.6 & 0.214 \\
\hline
\end{tabular}




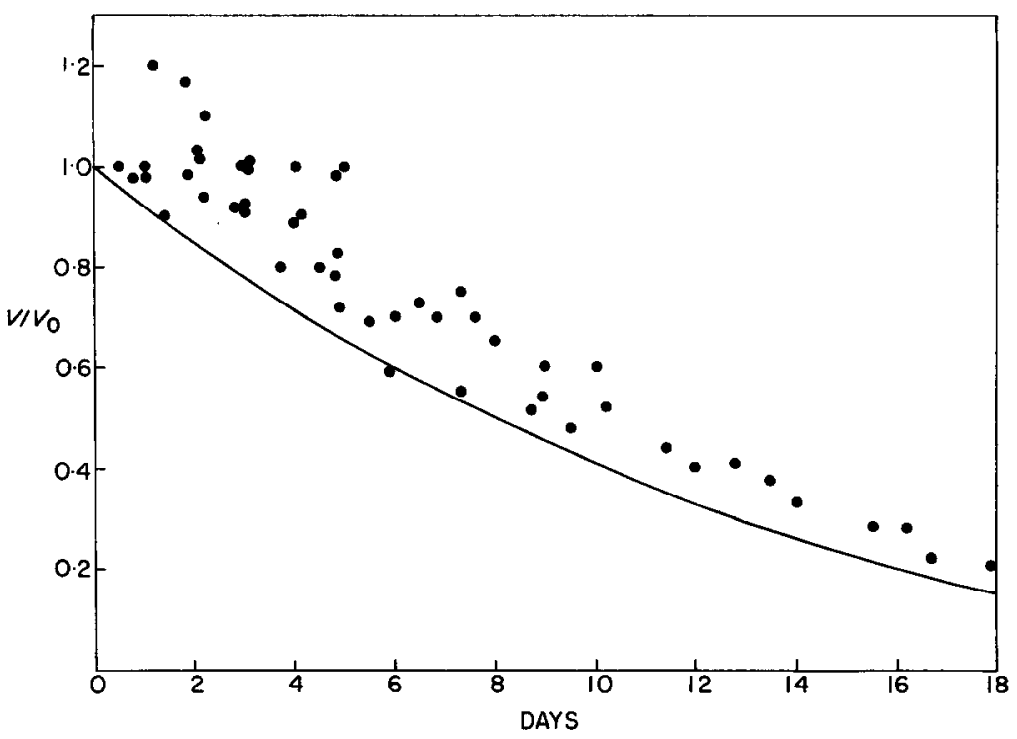

Fig. 1. Time course of nitrogen gas in a subcutaneous pocket of an airbreathing rat.

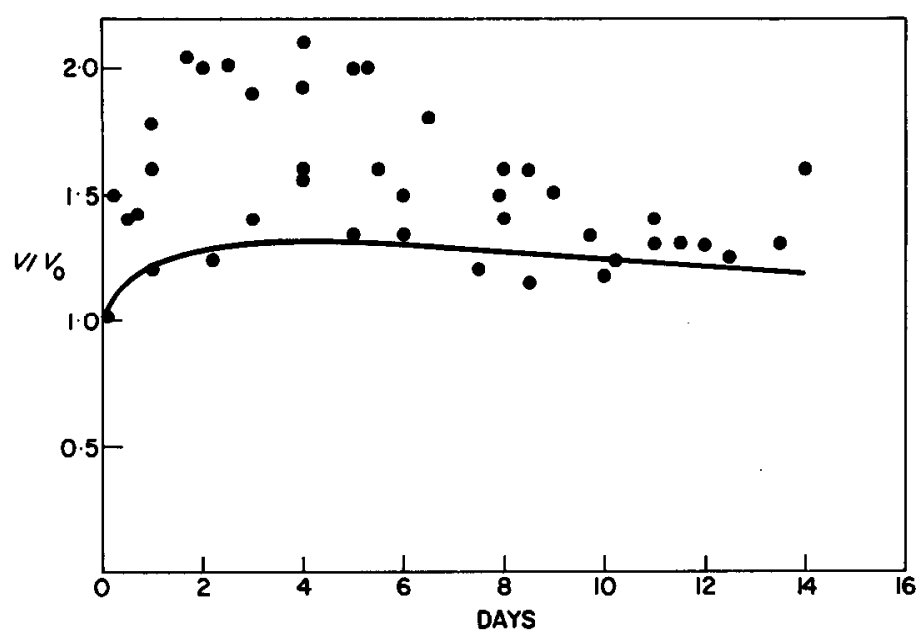

Fig. 2. Time course of sulfur hexafluoride gas in a subcutaneous pocket of an air-breathing rat.

saturation, is taken from Fig. 12 of Van Liew (1968a). The gas solubility in blood $\alpha_{b}$ is equal to that in tissue $\alpha_{t}$. These results were presented in Figs. 1-6 for comparison with the experimental data of Tucker and Tenney (1966). The ordinate represents the relative volume (the ratio of the instantaneous volume $V$ to the initial volume $V_{o}$ ). The solid lines are theoretical curves obtained by plotting equation (25) in the case of nitrogen gas or (29) in the cases of other inert gases. The experimental points are indicated as solid dots. In the calculations the total pressure inside the gas pocket has been assumed to remain at one atmosphere. The contribution of fluxes of $\mathrm{O}_{2}, \mathrm{CO}_{2}$ and $\mathrm{H}_{2} \mathrm{O}$ has been 


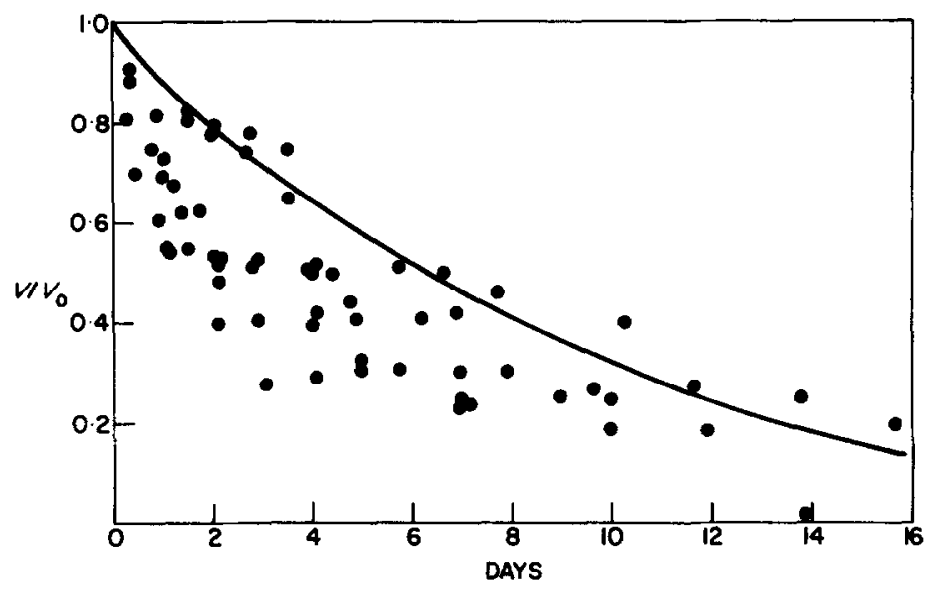

Fig. 3. Time course of helium gas in a subcutaneous pocket of an air-breathing rat.

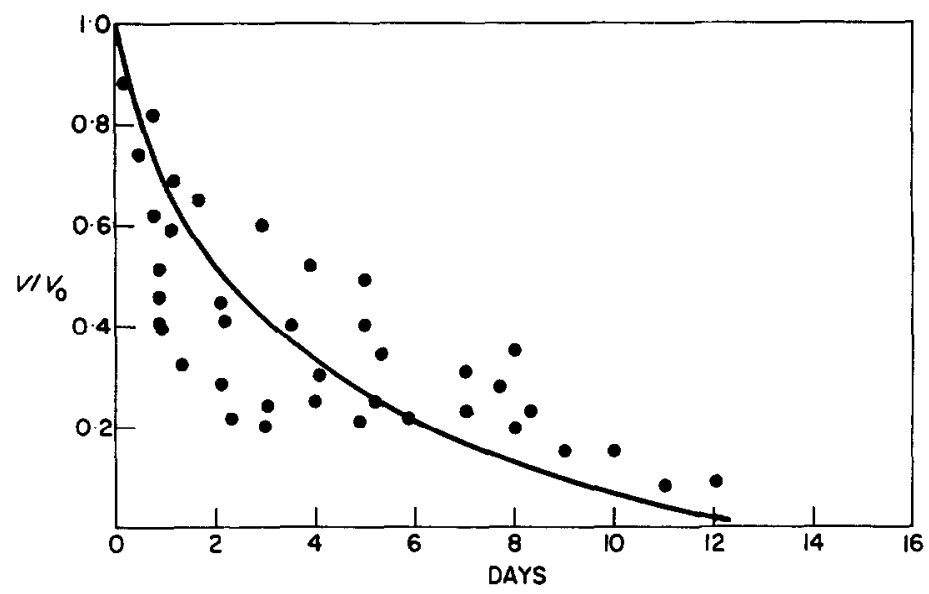

Fig. 4. Time course of argon gas in a subcutaneous pocket of an air-breathing rat.

neglected because these three gases only occupy 11 per cent of the total volume (Tucker and Tenney, 1966). Several different inert gas pockets are compared in the figures: a nitrogen gas pocket exemplifies the singlegas diffusion case. $\mathrm{SF}_{6}$ gas is nearly insoluble and represents the diffusion-controlling exchange process. On the other hand, $\mathrm{N}_{2} \mathrm{O}$ gas is highly soluble and represents the perfusion-controlling exchange process. The exchange process for argon, hydrogen or helium is both diffusion and blood flow determined.
It is seen in the figures that the agreement between theory and experiment is very satisfactory for all cases. The validity of the mathematical model which describes the mechanics of unsteady gas exchange between a subcutaneous gas pocket and the tissuecapillary system is thus confirmed.

\section{CONCLUDING REMARKS}

The theoretical analysis presented herein is based on a logical classification of dissolution of subcutaneous gas pockets or bubbles, according to its mechanism, into three 


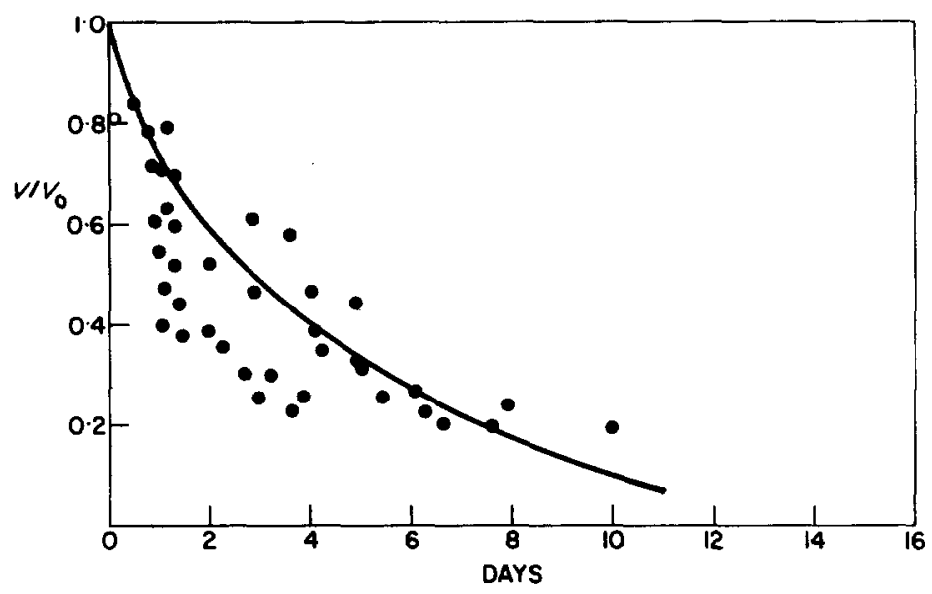

Fig. 5. Time course of hydrogen gas in a subcutaneous pocket of an air-breathing rat.

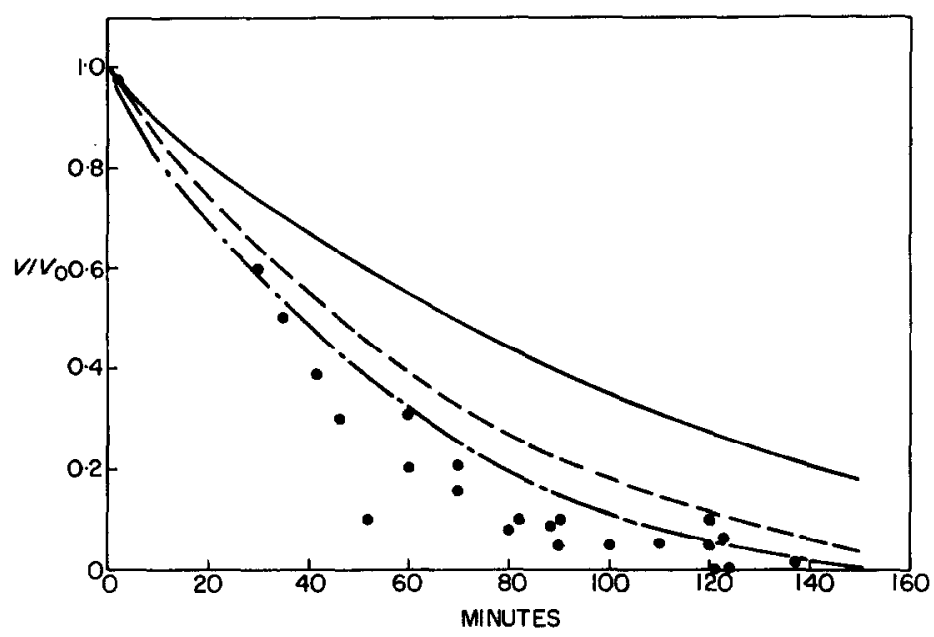

Fig. 6. Time course of nitrous oxide gas in a subcutaneous pocket of an air-breathing rat (from top $\alpha \times 10^{5}=68 \cdot 1,68 \cdot 1,120 ;(v \times 10=$ $4 \cdot 35,4 \cdot 35 ; 4 \cdot 35 ; k Q=0 \cdot 214,0 \cdot 50,0 \cdot 214)$.

categories: (i) tissue creep controlled, (ii) mass transfer controlled and (iii) the intermediate case where both effects are of importance. The special cases of (ii) with the neglect of the unsteady diffusion term and thermodynamic effects of the pocket gas reduce to the work of Van Liew (1968b).

The good agreement between the theoretical results of case (ii) and the experimental data of Tucker and Tenney (1966) indicates the general validity of the mathematical model which accounts reasonably well for both physical and physiological factors affecting gas exchange between the subcutaneous gas pocket and the tissue-capillary system.

The theory may be applied to determine the influx and efflux of gases across the surface of a subcutaneous pocket or a decomposition bubble, the tension of the dissolved gases in the tissues in both the unsteady and constant 
composition states, and the permeation of the dissolved gases in the tissues and their interaction with hemoglobin.

\section{REFERENCES}

Bartels, H. and Optiz, E. (1958) Handbook of Respiration (Edited by D. S. Dittmer and R. M. Grebe). Tables 12, 13 and 14. Saunders, Philadelphia.

Buckles, R. G. (1968) The physics of bubble formation and growth. Aerospace Med. 40, 1062-1069.

Carslaw, H. S. and Jaeger, J. C. (1959) Conduction of Heat in Solids, p. 63. Oxford University Press, London.

Chan, K. S. and Yang, W. J. (1969) Survey of literature related to the problems of gas embolism in human body. J. Biomechanics 2, 299-312.

Dale, W. A. and Rahn, H. (1952) Rate of gas absorption during atelectasis. Am. J. Physiol. 170, 606-615.

Epstein, P. S. and Plesset, M. S. (1950) On the stability of gas bubbles in liquid-gas solutions. J. chem. Phys. 18, 1505-1509.

Frankel, V. H. and Burstein A. H. (1970) Orthopaedic Biomechanics. Philadelphia, Lea and Febiger.

Harvey, Z. N., Barnes, D. K., McElroy, W. D., Whiteley, A. H., Pease D. C. and Cooper, K. W. (1944a). Bubble formation in animals-I. J. cell. Comp. Physiol. 24, $1-22$.

Harvey, Z. N., Whiteley, A. H., McElroy, W. D., Pease, D. C. and Barnes D. K. (1944b) Bubble formation in animals - II. J. cell. Comp. Physiol. 24, 23-34.

Harvey, Z. N. (1945) Decompression sickness and bubble formation in blood and tissues. Bull. N.Y. Acad. Med. 21, 505-536.

Krogh, A. (1919) The rate of diffusion of gases through animal tissues with some remarks on the cofficient of invasion. J. Physiol. Lond. 52, 391-408.

Piiper, J., Canfield, R. E. and Rahn, H. (1962) Absorption of various inert gases from subcutaneous gas pockets in rats. J. appl. Physiol. 17, 268-274.

Piiper, J., Humphrey H. T. and Rahn H., (1962) Gas composition of pressurized perfused gas pockets and fish swim bladder. J. appl. Physiol. 17, 275-282.

Piiper, J. (1963) Oxygen exchange of subcutaneous gas cavities in rats. Am. J. Physiol. 205, 1005-1007.

Rahn, H. and Canfield R. E. (1955) Volume changes and and the steady state behavior of gas pockets within body cavities. Wright Air Development Center Tech. Rep. No, 55-357, 395-408.

Tobin, C. E., Van Liew, H. D. and Rahn, H. (1962) Reaction of the subcutaneous tissue of rats to inspected air. Proc. Soc. exp. Biol. Med. 109, 122-126.

Tucker, R. W. and Tenney, S. R. (1966) Inert gas exchange in subcutaneous gas pockets of air-breathing animals: theory and measurement. Respirat. Physiol. 1, 151-171.

Van Liew, H. D. (1955) Volume and gas composition changes of subcutaneous gas pockets immediately following the injection of various gas mixtures. Wright Air Development Center Tech. Rep. No. 55-357, 409-414.

Van Liew, H. D. (1962a) Tissue gas tension by microtonometry; results in liver and fat. $J$. appl. Physiol. 17, 359-363.
Van Liew, H. D. (1962b) Oxygen and carbon dioxide permeability of subcutaneous pockets. Am. J. Physiol. 202, 53-58.

Van Liew, H. D. (1962c) Tissue $p \mathrm{O}_{2}$ and $p \mathrm{CO}_{2}$ estimation with rat subcutaneous gas pockets. J. appl. Physiol. 17, 851-855.

Van Liew, H. D., Bishop, B., Walder, D. P. and Rahn, H. (1965) Effects of compression on composition and absorption of tissue gas pockets. J. appl. Physiol. 20, 927-933.

Van Liew, H. D. and Passke, M. (1967) Permeation of neon, nitrogen and sulfur hexfluoride through walls of subcutaneous gas pockets in rats. Aerospace Med. 38, 829-831.

Van Liew, H. D. (1968a) Coupling and diffusion and perfusion in gas exit from subcutaneous pocket in rats. Am. J. Physiol. 214, 1176-1185.

Van Liew, H. D. (1968b) Interaction of $\mathrm{CO}$ and $\mathrm{O}_{2}$ with hemoglobin in perfused tissue adjacent to gas pockets. Respirat. Physiol. 5, 202-210.

Van Liew, H. D., Schoenfisch, W. H. and Olszowka, A. J. (1968/69) Exchanges of $N_{2}$ between a gas pocket and tissue in a hyperbaric environment. Respirat. Physiol. 6, 23-28.

Van Liew, H. D. and Hlastala, M. P. (1969) Influence of bubble size and blood perfusion on absorption of gas bubbles in tissues. Respirat. Physiol. 7, 111-121.

Yamada, H. (1970) Strength of Biological Materials (Edited by F. G. Evans). Baltimore, Williams and Wilkins.

Yang, Wen-Jei (1971) Dynamics of gas bubbles in whole blood and plasma. J. Biomechanics 4, 119-125.

Yang, Wen-Jei and Liang, C. Y. (1971) Behavior of gas bubbles in viscoelastic materials in a creep process. Submitted to J. appl. Phys.

\section{NOMENCLATURE}

$A$ constant defined as $\alpha_{b} k Q / \alpha_{t}$

$B$ constant defined as $q / \alpha_{t}$

$\Delta C$ change of dissolved gas content in blood

$D$ differential operator $\mathrm{d} / \mathrm{d} t$

$\mathscr{D}$ diffusion coefficient in tissue-capillary system

$H \quad \alpha_{t} / \rho$

$I_{i}$ function as defined by equation (18)

$i$ integer

$j$ integer

$k$ coefficient of end-capillary saturation

$m$ mass of gas inside bubble; $m_{0}$, initial value; $m_{i}$, of $j$-th component gas; $m_{o j}$, initial value of $m_{j}$

$P$ tension (pressure) of dissolved gas in tissuecapillary system; $P_{\infty}$, in capillary blood

$P_{g} \quad$ gas pressure inside bubble; $P_{g o}$, initial value; $P_{g j}$, of $j$-th component gas

$Q$ rate of actual blood perfusion per unit tissue volume

$q$ rate of oxygen consumption per unit tissue volume

$r$ radial distance in spherical coordinates as measured from the center of bubble or pocket

$R$ mean radius of bubble or pocket; $R_{o}$, initial value

$\bar{R}$ gas constant; $\bar{R}_{j}$, of $j$-th component gas

$T$ temperature of bubble and tissue-capillary system

$t$ time

u $r\left(P-P_{x}\right)$

$v_{r}$ radial component of the deformation rate tensor $\vec{v}$ 


\section{Greek letters}

$\alpha$ solubility of gas; $\alpha_{b}$, in blood; $\alpha_{t}$, in tissue

$\epsilon_{r r}$ strain; $\epsilon_{r r o}$, initial value; $\epsilon_{r r}$, creep rate

$\eta$ rheological constant of tissue-capillary system

$\theta$ angle in spherical coordinates

$\lambda$ rheological constant of tissue-capillary system

$\mu$ rheological constant of tissue-capillary system

$\rho$ density of gas inside bubble

$\sigma$ coefficient of surface tension

$\tau$ shear stress; $\tau_{r r}, \tau_{\theta \theta}$, and $\tau_{\phi \phi}$, normal components in the $r, \theta$, and $\phi$ direction, respectively; $\tau_{\infty},=\tau_{r r}(\infty, t)$ at a large distance from bubble; $\tau_{\text {rro }}$, initial value of $\tau_{r r}$

$\phi$ angle in spherical coordinates

\section{Superscript}

- time derivative

\author{
Subscripts \\ $b$ in capillary blood \\ $g$ gas inside bubble or pocket \\ $i$ integer; 1, for single-gas diffusion case; 2 , for \\ multi-gas diffusion case \\ $j$ j-th component gas inside bubble or pocket \\ $t$ in tissue \\ 0 at zero time or initial value \\ $\infty$ at a large distance from bubble
}

